\title{
DERECHO Y CIENCIA: \\ LA RACIONALIDAD CIENTÍFICA COMO FUNDAMENTO DE LA DECISIÓN JUDICIAL EN EL ORDENAMIENTO JURÍDICO COLOMBIANO
}

\author{
Olga Carolina Cárdenas Gómez ${ }^{1}$ \\ Milton CÉsar Jiménez Ramírez ${ }^{2}$
}

\begin{abstract}
RESUMEN: El objetivo de este artículo es describir el rol atribuido a la ciencia en la toma de decisiones judiciales en el ordenamiento jurídico colombiano. El aborda las funciones que está llamado a jugar el conocimiento científico en la toma de decisiones, con el fin de identificar la relación entre el derecho y la ciencia. Posteriormente, explica por qué el derecho ha perdido autonomía frente a la ciencia. Finalmente, analiza cómo la relación entre ciencia y derecho ha caído en el determinismo biológico en materia de pruebas de ADN. Por último, se ofrecerán las conclusiones sobre la necesidad de fomentar un reconocimiento de la racionalidad jurídica y las acciones para un trabajo con las ciencias.
\end{abstract}

Palabras clave: Ciencia, Derecho, valoración probatoria, filiación, prueba de ADN.

ABSTRACT: This article describes the role of science in Colombian judicial decision-making processes. It depicts the role that scientific knowledge plays in decision-making. This article allows us to identify the way in which law and science relate. It then explains why science has made the law lose autonomy, and how the relationship between science and law has fallen into biological determinism in the matter of DNA tests. This article concludes that there is a need to promote the recognition of legal rationality and refers to some actions that allow working with science.

Keywords: Science, Law, evidentiary assessment, filiation, DNA test.

\section{INTRODUCCIÓN}

El conocimiento científico contribuye a la toma de decisiones a través de la experticia o peritaje desde hace más de un siglo ${ }^{3}$. Gracias a su constante evolución y a características como la certeza y objetividad, otras disciplinas de corte más social como el derecho y la ciencia política lo aplican al margen de su propia racionalidad. Hemos caído en un

1 Doctora en derecho, Universidad de Laval, Canadá. Docente investigadora del Departamento de Jurídicas, Universidad de Caldas (Colombia). Código Orcid: 0000-0002-4448-2449. Dirección Postal: Calle 65 26-10, Manizales, Caldas (Colombia). Dirección electrónica: carolina.cardenas@ucaldas.edu.co. El trabajo es resultado del proyecto de investigación denominado: "El Derecho y la Ciencia. La racionalidad como fundamento de la decisión judicial”, del que es investigadora responsable.

2 Doctor en derecho, Universidad Carlos III de Madrid, España. Docente investigador, del Departamento de Jurídicas, Universidad de Caldas (Colombia). Código Orcid: 0000-0003-1149-4723. Dirección Postal: Calle 65 26-10, Manizales, Caldas (Colombia). Dirección electrónica: milton.jimenez@ucaldas.edu.co. El trabajo es resultado del proyecto de investigación denominado: "El Derecho y la Ciencia. La racionalidad como fundamento de la decisión judicial”, del que es investigador responsable.

3 JaSANOFF (1987) p. 200. 
determinismo científico dada la posibilidad que ofrecen los conocimientos científicos de producir resultados contundentes. En derecho esta situación puede evidenciarse al estudiar la evolución que las contribuciones científicas han tenido en los procesos de filiación. Antes de la prueba de $\mathrm{ADN}$ era común que la paternidad o maternidad se decidiera recurriendo a medios probatorios imprecisos o lejanos (declaraciones y confesiones), distantes de ofrecer claridad metodológica respecto a su atribución.

De 1968 a 2001, la ciencia pasó de ser una herramienta para ayudar a la toma de decisiones, a ser el mecanismo que señala la decisión a tomar. La ciencia determina quién es padre o madre. El juez delega entonces su función de valorar las pruebas y tomar una decisión para ratificar la información científica a través de una sentencia judicial. Ante esa realidad, surge la pregunta: ¿cuál es la relación entre el derecho y la ciencia al momento de emplearla como fundamento de la decisión judicial? La hipótesis de este trabajo es que se trata de una relación donde el derecho se subordina a la ciencia a pesar de tratarse de sistemas autónomos y del deber del juez, como director del proceso, de preservar la virtud de la sana crítica al momento de emplear pruebas científicas con el fin de distinguir entre verdad material y verdad procesal, que es la que se busca respetar y proteger con la decisión judicial.

Para demostrar la hipótesis, el trabajo se dividirá en tres partes: primero, se presentarán los modelos sobre la función del conocimiento científico en la toma de decisiones para identificar en qué modelo se ubica la relación entre el derecho y la ciencia. Segundo, se explicará cómo el derecho ha perdido autonomía frente a la ciencia en virtud de la ausencia de un lenguaje común que regule su relación. Esta ausencia ha permitido la prevalencia de la ciencia sobre el derecho, determinando la forma en que los hechos se consideran probados dentro del proceso judicial. Finalmente, se expondrá la evolución de la relación entre la ciencia y el derecho, que ha pasado de la autonomía al determinismo biológico, dado que los jueces asumen los resultados de la prueba de ADN como verdad procesal absoluta. Por último, a partir del análisis sobre la relación entre la ciencia y el derecho, se evidenciará la necesidad de fomentar un reconocimiento de la racionalidad jurídica y el establecimiento de un trabajo cooperativo con la ciencia.

Para llegar a los resultados se empleó la investigación documental, con enfoque crítico y hermenéutico. La identificación de la documentación pertinente se realizó a través de diferentes bases de datos jurídicas. La información se concentró principalmente en temas sobre la relación entre ciencia y derecho, la teoría de la prueba y la toma de decisiones en los procesos de filiación. Para la recolección y análisis de la información se emplearon fichas bibliográficas que permitieron organizar la información en forma sistemática.

\section{EL CONOCIMIENTO CIENTÍFICO EN LA TOMA DE DECISIONES}

El conocimiento científico proporciona a los responsables de la toma de decisiones un panorama de lo que está en juego y un inventario de las soluciones posibles para atender una situación particular que exige una decisión. De ahí que BECK afirme que la racionalidad de la ciencia siempre ha sabido encontrar soluciones a todos los problemas ${ }^{4}$. Los cono-

\footnotetext{
4 BECK (2008) p. 83.
} 
cimientos científicos se convierten entonces en una herramienta que legitima las decisiones en contextos caracterizados por la incertidumbre.

La utilización del conocimiento científico en la toma de decisiones implica distinguir dos momentos: el de la racionalidad que corresponde al saber, y el del juicio de valor que corresponde al poder ${ }^{5}$. El científico o el experto entrega su experticia a una autoridad competente para que esta tome la mejor decisión posible en un contexto determinado, según las características propias de la situación que intenta intervenir o resolver ${ }^{6}$ (ver gráfica 1 ).

\section{GRÁFICA 1}

El conocimiento científico en la toma de decisiones

Proceso de toma de decisiones

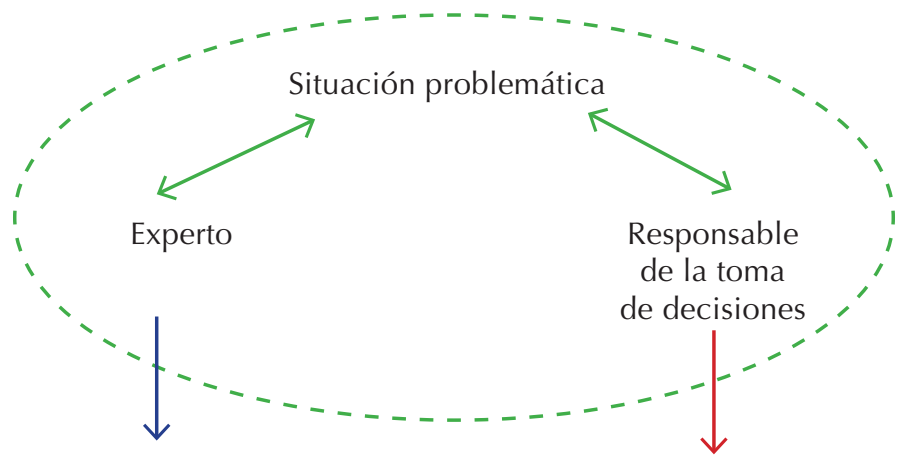

Hacer constataciones Mencionar lo que esta en juego Hacer un inventario de las opciones posibles
Evaluar las recomendaciones de los expertos

Tomar decisiones imparciales, transparentes y objetivas

La relación entre saber y poder puede explicarse a través de dos modelos: el decisional o subjetivo y el tecnocrático o racional ${ }^{7}$. En el primero, los responsables de la toma de decisiones recurren al peritaje, como mecanismo instrumental para "legitimar" una decisión previamente tomada en la esfera del poder ${ }^{8}$. Este modelo es conocido como "politización del científico"' (ver gráfica 2). En este modelo, el conocimiento científico permite sustentar las decisiones como racionales y pertinentes para garantizar la obtención de los resultados buscados ${ }^{10}$.

\footnotetext{
EWALD (1992) p. 204.

6 Encinas de Munagorri (2002) p. 380.

7 Un tercer modelo conocido como modelo pragmático no será empleado en el desarrollo de este artículo.

8 Hassenteufel (2008) p. 202.

9 Weingart (1999) p. 156. Doering (2010) p. 1.

10 Pauvert (2006) p. 271.
} 


\section{GRÁFICA 2}

El conocimiento científico en el modelo decisional

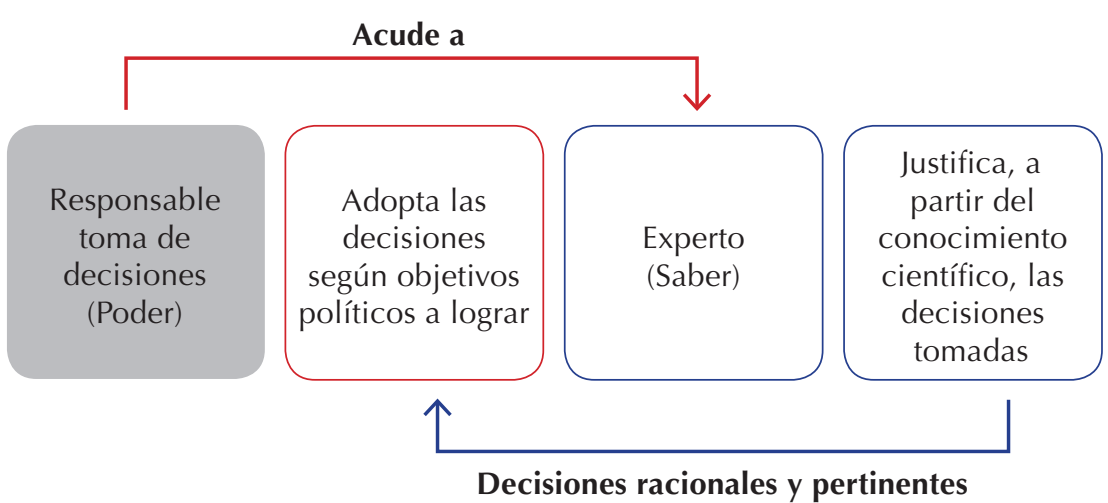

No obstante, la relación entre poder y saber se invierte en el modelo tecnocrático. En este modelo, los expertos salen del campo del saber para instalarse en el campo del poder abrogándose la capacidad de tomar decisiones ${ }^{11}$. Este modelo puede ser representado por la expresión "la confiscación del poder por el saber"12 o "cientifización del poder"13 (ver gráfica 3).

\section{GRÁFICA 3}

El conocimiento científico en el modelo tecnocrático

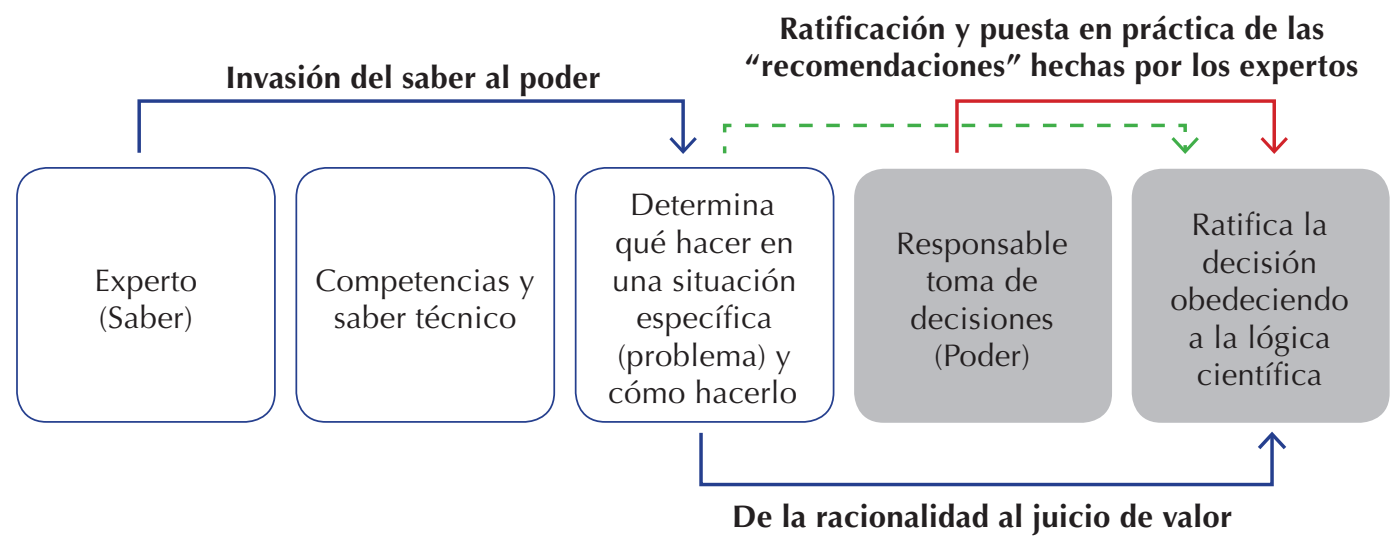

El modelo tecnocrático contraría la división del trabajo propia de la racionalidad científica. En efecto, la invasión del saber al campo del poder contraría los postulados sobre la diferenciación funcional de Luhmann ${ }^{14}$. Según BECK, la ciencia va más allá de su frontera porque ella no puede hacer valer la autoridad que le confiere su racionalidad al momento

\footnotetext{
11 Meynaud (1957) p. 27.

12 JaCOB y Genard (2004) p. 9.

13 Weingart (1999) p. 154. Doering (2010) p. 1.

14 LuHMann (2007) p. 97.
} 
de hacer juicios de valor. En consecuencia, la actividad propiamente política de la toma de decisiones desaparece bajo la ilusión de argumentos objetivos y controlados por los especialistas ${ }^{15}$. Esta situación refleja la relación entre el derecho y la ciencia en materia de procesos de filiación.

\section{EL DERECHOY SU PÉRDIDA DE AUTONOMÍA FRENTE A LA CIENCIA}

La diferenciación funcional generada por procesos como la globalización, la democratización y el pluralismo originó sistemas sociales especializados como la economía, el derecho, la política o la ciencia, caracterizados por una alta contingencia y una creciente complejidad ${ }^{16}$. Esos sistemas operan según sus propias lógicas a través de actores y códigos. Dichos códigos son únicos, ya que ningún otro sistema los utiliza, y en caso de hacerlo, difícilmente puede comprenderlos o aplicarlos a su propia racionalidad.

La función de cada sistema se especializa logrando resolver problemas que otros no pueden ${ }^{17}$. Esto se explica según Maturana y Varela porque los sistemas son cerrados operacionalmente y determinados estructuralmente ${ }^{18}$. El derecho se especializa en decisiones fundadas en la correcta valoración de las normas y las pruebas; en la averiguación de la verdad sobre lo ocurrido y en la correcta aplicación del derecho sustantivo ${ }^{19}$. El derecho también se especializa en la creación de la norma como su objeto; en la imputación de toda norma frente a una norma hipotética fundamental o superior ${ }^{20}$; o desde la reflexión teórica de qué es el derecho, en la norma y sus clasificaciones (normas primarias y secundarias) ${ }^{21}$.

Dado que las funciones de cada sistema son diferentes, éstos son autónomos, ya que cada sistema asume una posición para producir y reproducir las operaciones de la sociedad dentro de su campo de acción. Los sistemas autónomos pueden mostrarse "cognitivamente abiertos a su entorno sobre la base de sus propias distinciones" ${ }^{22}$. Para garantizar la probabilidad de éxito de cualquier interacción es necesario que el sistema intervenido acepte la intervención a través de una invitación que le permita mejorar la comprensión o contribuir a la solución de un problema que no puede resolver correctamente y de forma independiente. Dicha invitación permite que una intervención se instale en otro sistema como un regalo con sentido ${ }^{23}$. La integración de este regalo se hace teniendo en cuenta la lógica del sistema al cual pertenece el observador, donde la referencia de lo observado o descrito siempre es el sistema mismo.

A partir de sus códigos y de sus reglas para resolver los conflictos, el Derecho ha aceptado la contribución de la ciencia. Sin embargo, como la ciencia tiene igualmente sus

\footnotetext{
5 Granjou (2003) p. 176.

16 Vallejos-Romero y Valdivieso (2014) pp. 323-324. Habermas et al. (1984) pp. 18-28.

17 Vallejos-Romero (2013) p. 5.

18 Maturana y Varela (1984) p. 6.

19 Taruffo (2006) pp. 253-277 y Taruffo (2008) pp. 131-140. Ferrer (2013) pp. 22-24.

20 Kelsen (1995) pp. 3-6 y 17-23.

21 HaRT (1963) pp. 33-53.

22 Mascareño (2011) p. 2.

23 Vallejos-Romero y Valdivieso (2014) p. 330.
} 
códigos y reglas, resulta necesario identificar el lenguaje común que estas disciplinas crean para comunicarse sin perder su autonomía. Con la prueba científica, el derecho incluye un elemento de la ciencia y reconoce los aportes que ella puede hacer dentro de las condiciones de su propia clausura operativa. Empero, la ausencia de un lenguaje común impide que esta relación se caracterice por la autonomía de los sistemas. La relación entre estos sistemas está entonces siendo dominada por la ciencia debido a su claridad racional interna, a las dificultades de los sistemas jurídicos para asimilar las complejidades de los hechos sujetos a su análisis y a las contribuciones e incidencias de otros sistemas ${ }^{24}$.

Los productos que la ciencia ofrece al derecho se caracterizan por la contundencia práctica de sus resultados, lo que ha conllevado al desplazamiento del conocimiento jurídico, que a la par, no posee consenso sobre su carácter científico, es decir, su estructura racional, objeto y la autonomía de sus resultados ${ }^{25}$.

En materia de filiación, esta relación ha cambiado. De una relación autónoma, pasamos a una relación de subrogación. La relación autónoma puede evidenciarse en la Ley No 75 de 1968, que reconoció por primera vez la contribución de la ciencia a través de las pruebas antropo-heredo-biológicas ${ }^{26}$. En los procesos de filiación, dicha prueba debía valorarse junto con otras, dada la subjetividad que conllevaba comparar las características físicas entre dos personas y la alta probabilidad de encontrar individuos con las mismas características.

En 2001, la adopción de la Ley No 721 cambió la relación de autónoma a subordinada, debido a la exigencia de practicar, en todos los procesos de filiación, "exámenes que científicamente determinen índice de probabilidad superior al $99.99 \%$ " 27 . Para reafirmar esta subordinación, la ley estableció que "[s]ólo en aquellos casos en que es absolutamente imposible disponer de la información de la prueba de $\mathrm{ADN}$, se recurrirá a las pruebas testimoniales, documentales y demás medios probatorios para emitir el fallo correspondiente" 28 . La subordinación se mantuvo en la Ley No 1564 de 2012, que establece que "cualquiera que sea la causal alegada, en el auto admisorio de la demanda el juez ordenará aún de oficio, la práctica con marcadores genéticos de $\mathrm{ADN}$ o la que corresponda con los desarrollos científicos y advertirá a la parte demandada que su renuencia a la práctica de la prueba hará presumir cierta la paternidad, maternidad o impugnación alegada" 29 .

La prueba de $\mathrm{ADN}$ determina el vínculo biológico con una certeza que el derecho ha deseado, pero no ha logrado alcanzar. De manera crítica, GASCÓN ${ }^{30}$ estima que las pruebas científicas han estado acompañadas de un aura de infalibilidad que ha impedido cualquier reflexión sobre las mismas, pues su validez y valor probatorio se han asumido como un dogma de fe, lo que paradójicamente es contrario a la metodología científica. Además, TARUFFO $^{31}$ ha señalado que la certeza e infalibilidad de la ciencia son solo un mito, porque

24 Allen (2013) pp. 52-60.

25 Von-Kirchmann (1949) pp. 40 -49.

26 Artículo 7o Ley No 75 de 1968.

7 Artículo 1º Ley No 721 de 2001.

Artículo 30 Ley No 721 de 2001.

Artículo 386, Ley No 1564 de 2012.

GASCÓN (2012) pp. 108-122.

31 Taruffo (2006) pp. 281-293. 
a pesar de influenciar los estados mentales del juez y proporcionarle informaciones controladas y fiables, ella no siempre contribuye al descubrimiento de la verdad.

En la actualidad, la subordinación es explícita e implícita ${ }^{32}$. La explícita se funda en la exigencia legal de recurrir a la ciencia, no solo como un criterio para integrar el acervo probatorio, sino como una prueba (procesal y material) contundente, capaz de definir aquello que el juez debe decidir, dado que el resultado de la prueba permite establecer dos hechos: (i) El supuesto padre o madre demandado transmitió sus caracteres genéticos a quien busca establecer su filiación y (ii) las relaciones sexuales entre las personas que se encuentran vinculadas al proceso (generalmente, madre y supuesto padre, si bien otras configuraciones pueden presentarse) dentro del periodo en el que la ley presume la concepción. No obstante ¿se adecúan estos dos hechos completamente a los supuestos de la norma que busca determinar quién es padre? Con una consideración adicional y es el actual reconocimiento de la voluntad procreacional como fuente de atribución de la filiación.

Por su parte, la subordinación implícita implica aceptar que la prueba científica, dadas sus características, permite al juez cumplir con la pretendida convicción necesaria para alcanzar la verdad formal, que constituye una promesa de lo procesal ${ }^{33}$. Además, y en eso radica su creciente carácter incontrovertible, la consideración de que puede entregar la verdad material, como pasa en los casos de filiación. Al respecto, debe señalarse que esta posición deja completamente al margen la distinción entre verdad material, que determina quién transmitió los caracteres genéticos a una persona, y la verdad procesal que nos permite determinar quién debe ser declarado padre o madre en un proceso de filiación.

La pretendida contundencia de la prueba de ADN está entonces llevando al juez a caer en un determinismo científico y al Derecho a perder su autonomía. En efecto, el resultado está determinando la decisión por dos razones: (i) los principios científicos en los que se funda la prueba, y (ii) la imposibilidad de contradecirla cuando el resultado arroja una probabilidad de paternidad de 99,99\%. Además, el conocimiento científico genera en el juez la prevalencia de los principios de celeridad y tutela efectiva, pues al estar convencido de que llegó a la verdad, solo le queda proferir una sentencia bajo el supuesto de que ha acertado y que su proveído reviste de legalidad una relación jurídica que estuvo carente de ella.

Este desplazamiento de la jurisdicción por la ciencia supone una subordinación del derecho, pues el operador jurídico optó por decretar la prueba sin mayor análisis sobre los hechos particulares de cada caso con el fin de no desconocer la ley y, al momento de valorarla, la ratifica si el resultado es superior al 99,99\%, sin verificarlo y contradecirlo bajo un método crítico y respetuoso de los sistemas en interacción. El regalo que la ciencia hace al derecho carece entonces de sentido, ya que el juez no construye una comunicación entre su sistema y el sistema "ciencia", sino que permite que un sistema con reglas y códigos extraños al suyo resuelva los problemas que le son propios. Al renunciar a la práctica argumentativa y al análisis normativo, no es el juez quien decide, sino la ciencia. En esa medida el juez es un simple observador del proceso cuya función, en este caso en particular, se reduce a ratificar la información científica tal y como se evidenció en el modelo tecnocrático.

32 TARuffo (2006) pp. 303-317.

33 Ferrer (2005) pp. 55-73. 


\section{LA RELACIÓN ENTRE LA CIENCIA Y EL DERECHO EN LA DETERMINACIÓN DE LA FILIACIÓN}

Las pruebas científicas empleadas en los procesos de filiación han sido las pruebas antropo-heredo-biológicas, los grupos sanguíneos, las proteínas séricas, los antígenos de histocompatibilidad y los marcadores de ADN. Las tres primeras pruebas se caracterizaban por tener un alto poder de exclusión, pero un bajo poder de inclusión ${ }^{34}$. En consecuencia, la forma como estas pruebas eran valoradas por los jueces dependía de diversos factores, como: (i) la claridad sobre los principios que regían su práctica y su fundamento biológico; (ii) el culto a la exégesis que no permitía fallar de acuerdo con la realidad biológica en ausencia de pruebas o de una norma que así lo ordenara y (iii) la prueba científica solo adquiría certeza en la medida en que otros medios probatorios obraran en el proceso ${ }^{35}$. Entre 1968 y 1996, aproximadamente, los jueces consideraron suficiente la prueba científica para excluir la maternidad o paternidad, pero no para atribuirla. En esa medida, aportar otras pruebas al proceso para lograr el convencimiento del juez era absolutamente necesario.

En 1996, empiezan a realizarse pruebas con antígenos de histocompatibilidad, las cuales ofrecen un poder de inclusión superior al $98 \%{ }^{36}$. Si bien estas pruebas permitían determinar, a partir de los predicados de Hummel ${ }^{37}$, que la paternidad es muy probable, los jueces insistían en que el resultado no era suficiente para establecer la maternidad o paternidad ${ }^{38}$. A partir de 1997 , la prueba de $\mathrm{ADN}$ fue estandarizada por los laboratorios colombianos y empezó a practicarse dentro de los procesos de filiación. Esta prueba alcanza probabilidades de inclusión de 99,99\%, lo que según Hummel ${ }^{39}$ permite considerar la paternidad prácticamente probada.

Si bien entre 1998 y 2001 la prueba fue practicada en los procesos de filiación, los jueces se negaron a atribuir la maternidad o paternidad en ausencia de otras pruebas. Los argumentos empleados por los jueces para negar la declaratoria de maternidad o paternidad fueron que la prueba de ADN no podía aceptarse como plena prueba y que para hacerlo era necesario promover una reforma legal que incluyera la prueba de $\mathrm{ADN}$ como causal independiente de investigación de la filiación ${ }^{40}$. Los jueces parecían no estar preocupados por asegurar una paternidad responsable, sino solamente disponer de un mecanismo para determinar, con un alto grado de certeza, quien debería ser declarado padre o madre equiparando proveedor biológico, es decir, quien transmite los genes, al padre o madre, entendido como quien ama, cuida y apoya. Esta argumentación no era aceptada unánimemente. Tribunales como el de Buga, Medellín y Bogotá consideraban que dicha modificación no era necesaria porque el resultado de la prueba de $\mathrm{ADN}$ permitía inferir que la madre y el pretendido padre sostuvieron relaciones sexuales durante los 120 días en que la ley presume

34 Huguet, Carracedo y Gené (1988) p. 86.

35 CÁrdenas et al. (2004) pp. 19 y 22-23.

36 Huguet, Carracedo y Gené (1988) p. 72.

37 Hummel (1971) p. 95.

38 Tribunal Superior de Distrito Judicial de Popayán. Sentencia del 15 de mayo de 1998.

39 Hummel (1971) p. 95.

40 Tribunal Superior de Distrito Judicial de Buga. Sentencia del 19 de mayo de 1998. 
la concepción ${ }^{41}$. Esta posición se explica porque el cálculo de probabilidad en las pruebas de $\mathrm{ADN}$ se hace a partir de frecuencias poblaciones que impiden encontrar otro hombre con la misma probabilidad ${ }^{42}$. Uno de los magistrados del Tribunal Superior de Distrito Judicial de Buga señalaba que con pruebas de ese linaje los procesos de filiación cuentan con un elemento probatorio del cual emerge un mayor poder de convicción, dado que su eficacia normativa no se limita al campo de la compatibilidad, sino que se adentra en el campo de la probabilidad ${ }^{43}$. Esta posición, si bien se argumenta de una forma diferente, no dista mucho de la ya expuesta.

Después de la Ley No 721 de 2001 se han presentado casos donde el juez ha ordenado la prueba a pesar de la existencia de dudas sobre la calidad e integridad de la muestra, o cuando ha quedado claro, a partir de la narración de los hechos que sustentan el caso, que el padre o madre e hijos no comparten ningún vínculo genético. En estos casos, la práctica de la prueba de $\mathrm{ADN}$ se ha hecho con el fin de llegar a la verdad formal. No obstante, la prueba, aun debidamente practicada, puede arrojar resultados que no se compadecen con la verdad procesal, porque la muestra del supuesto padre o madre pudo ser cambiada o porque no existen vínculos genéticos entre quienes hacen parte del proceso. En esos casos, la pregunta que surge es ¿por qué decretar la prueba si ella no aportará información útil y pertinente al proceso?

\section{CONCLUSIONES}

El derecho y la ciencia, pese a su autonomía, mantienen relaciones de cooperación que permiten asegurar la certeza y pertinencia de las decisiones que se toman en cada uno de ellos. En esa medida, la valoración de la prueba de ADN dentro de los procesos de filiación no debe obedecer a un determinismo científico sino a una crítica razonable respetuosa del método científico, de los derechos fundamentales de los actores procesales y de otros medios probatorios según cada caso concreto. Ciertamente, la prueba puede resultar útil en casos donde la paternidad biológica coincide con la paternidad jurídica, pero su decreto en todos los casos de filiación es una clara muestra de subordinación del derecho a la ciencia

Si bien no puede desconocerse que la subordinación se justifica en la objetividad y reproductibilidad de los resultados científicos, en materia de filiación resulta necesario distinguir entre la paternidad biológica y la paternidad jurídica o legal. La valoración de las pruebas de $\mathrm{ADN}$ y su empleo en los procesos de filiación debe hacerse a partir de un replanteamiento sobre lo que el juez busca determinar: aunque la ciencia puede revelar quien es el proveedor genético, la verdad material, ella no siempre es la verdad jurídica que debe buscar el juez.

La pregunta que queda es: ¿cuáles son las características que deben regular la relación entre el derecho y la ciencia como sistemas de conocimiento autónomos? La construcción de una respuesta requiere revisiones de los fundamentos racionales, tanto de lo jurídico

\footnotetext{
41 Núm. 4o , Art. 6, Ley No 75 de 1968.

42 Cárdenas et al. (2004) p. 21.

43 Tribunal Superior de Distrito Judicial de Buga. Sentencia del 19 de mayo de 1998.
} 
como de lo científico, y análisis que se ocupen de los alcances de una práctica científica cada vez más reputada de la autonomía de la ciencia jurídica, todo con el propósito de construir márgenes de acción racionales y de trabajo cooperativo desde las diferencias y vacíos que una u otra disciplina pueden satisfacer.

\section{BIBLIOGRAFÍA CITADA}

Allen, Ronald (2013): “Los estándares de prueba y los límites del análisis jurídico”, en VÁzQuez, Carmen (Ed.). Estándares de prueba y prueba científica. Ensayos de epistemología jurídica (Barcelona, Marcial Pons).

BECK, Ulrich (2008): La société du risque. Sur la voie d'une autre modernité (Paris, Flammarion).

Cárdenas Gómez, Olga Carolina, Álvarez Rosero, Rosa Elvira y Muñoz Benítez, Sulma Lilian (2004): "Las pruebas genéticas en los procesos de filiación”, Revista de la Facultad Ciencias de la Salud, vol. 6, No 3: pp. 19-23.

Doering, Ronald L. (2005): "Politicizing Science; Scientizing Politics". Disponible en: https://www.lexology.com/library/detail.aspx?g=58dd6663-10fc-483d-9ac581cf37f71e84. Fecha de consulta 23 de enero de 2021.

Encinas de Munagorri, Rafael (2002): “Quel statut pour l'expert?”, Revue française d'administration publique, No 103: pp. 379-389.

Ewald, François (1992): "L'expertise une illusion necessaire", en THEYs, Jacques y KaLAORA, Bernard (dir.) La Terre outragée: les experts sont formels! (Paris, Autrement).

Ferrer, Jordi (2005): Prueba y verdad en el Derecho (Madrid, Editorial Marcial Pons).

FERRER, Jordi (2013): "La prueba es libertad, pero no tanto: Una teoría de la prueba cuasibenthamiana”, en VÁZQUeZ, Carmen (Ed.). Estándares de prueba y prueba científica. Ensayos de epistemología jurídica (Barcelona, Marcial Pons).

GASCÓN, Marina (2012): Cuestiones Probatorias (Bogotá, Universidad Externado de Colombia).

Granjou, Céline (2003): "L'expertise scientifique à destination politique", Cahiers Internationaux de Sociologie, No 114 : pp. 175-183.

Guerrero, Cesar Augusto (2010): "Filiación en Colombia: de la incertidumbre a la ciencia. Una recensión legal y jurisprudencia”, Pensamiento Jurídico, No 29: pp. 107-122.

Hart, Herbert (1963): El concepto del Derecho (Buenos Aires, Abeledo Perrot).

Habermas, Jürgen (1984): Ciencia y Técnica como Ideología (Madrid, Editorial Tecnos).

Hassenteufel, Patrick (2008): Sociologie politique: L'action publique (Paris: A. Colin).

Huguet, Emily; Carracedo, Ángel y Gené, Manuel (1988): Introducción a la investigación biológica de la paternidad (Barcelona, Promociones y Publicaciones universitarias S.A.).

Hummel, Konrad (1971): Biostatistical Opinion of Parentage (Suttgart, Gustav Fischer Verlag).

JaCOB, Steve y Genard, Jean-Loius (2004): "L'État et les savoirs experts: la production cognitive en mutation", en JАСOB, Steve y GENARD, Jean-Loius (edit.), Expertise et action publique (Bruxelles, Éditions de l'Université de Bruxelles) pp. 7-12.

JaSAnoff, Sheila (1987): "Contested Boundaries in Policy-Relevant Science", Social Studies of Science, vol. 17, No 2: pp. 195-230. 
Kelsen, Hans (1995): Teoría general del Derecho y del Estado (México, Universidad Nacional Autónoma de México).

LuHMAnN, Niklas (2007): La sociedad de la sociedad (México, Herder/Universidad Iberoamericana).

MascareÑo, Aldo (2011): "Sociología de la intervención: orientación sistémica contextual”, Revista Mad, vol. 25: pp. 1-33.

Maturana, Humberto y Varela, Francisco (1984): El árbol del conocimiento (Santiago de Chile, Organización de Estados Americanos (OEA)/Editorial Universitaria).

Meynaud, Jean (1957): "Les techniciens et le pouvoir", Revue française de science politique, No 7: pp. 5-37.

Pauvert, Bertrand (2006): "Expert, expertise et décision publique", Droit de l'environnement, No 142: pp. 270-275.

TARUfFO, Michele (2006): Sobre las fronteras. Escritos sobre la justicia civil (Bogotá, Editorial Temis).

Taruffo, Michele (2008): La Prueba (Barcelona, Marcial Pons).

Vallejos-Romero, Arturo (2013): "Conflictos riesgos e intervención socioambiental. Una aproximación sociológica”. Ponencia presentada en el XI Congreso Español; de Sociología, 10 al 12 de julio de 2013, Facultad de Ciencias Politicas y Sociología, Universidad Complutense de Madrid. Disponible en: https://studylib.es/doc/1814938/conflictos--riesgos-eintervenci\%C3\%B3n-socioambiental-una-apr.... Fecha de consulta 23 de enero de 2021.

Vallejos-Romero, Arturo y Valdivieso, Patricio (2014): "Hacia políticas públicas de segundo orden en América Latina”, Andamios, vol. 11, No 26: pp. 323-352.

Von Kirchmann, Julius Hermann (1949): La jurisprudencia no es ciencia (Madrid, Civitas).

Weingart, Peter (1999): "Scientific expertise and political accountability: paradoxes of science in politics", Science and Public Policy, vol. 26, No 3: pp. 151-161.

\section{NORMAS CITADAS}

Colombia, Ley No 75 de 1968 (30/12/1968), Por la cual se dictan normas sobre filiación y se crea el Instituto Colombiano de Bienestar Familiar.

Colombia, Ley No 721 de 2001 (29/12/2001), Por medio de la cual se modifica la Ley 75 de 1969.

Colombia, Ley No 1564 de 2012 (12/7/2012), Por medio de la cual se expide el Código General del Proceso y se dictan otras disposiciones.

\section{SENTENCIAS CITADAS}

Tribunal Superior de Distrito Judicial de Buga. Sentencia del 19 de mayo de 1998. Magistrado Ponente: Felipe Francisco Borda Caicedo. Sentencia no numerada.

Tribunal Superior de Distrito Judicial de Popayán. Sentencia del 15 de mayo de 1998. Magistrado Ponente: Ciro Sandoval. Sentencia no numerada. 
